\title{
Evaluation of Some Faba Bean Genotypes Under Three Planting Dates in Middle Egypt
}

\author{
S. M. Tarek*; Marwa Kh. A. Mohamed and Rehab A.M.A. \\ Food Legumes Research Department (FLRD), Field Crops Research Institute (FCRI), \\ Agricultural Research Center (ARC), Giza, Egypt. \\ *Tareksaber456@gmail.com
}

\begin{abstract}
The recent work was conducted in the Agricultural Research Stations of Mallawy, El-Minia Governorate and Sids, Beni-Suif Governorate, Egypt, during two successive winter seasons of 2016/2017 and 2017/2018 to study the effect of sowing date on productivity of eight faba bean genotypes, (Sakha-1, Sakha-3, Giza-843, Giza-716, Pop.-10, SB-1, BF-10 and Sids-19). Three sowing dates (October $15^{\text {th }}$, November $1^{\text {st }}$ and November $15^{\text {th }}$ ) were used. The results of the combined analysis of the two growing seasons 2016/2017 and 2017/2018 showed that, number of days to both flowering and maturity and number of branches were significantly affected by sowing date. The productivity traits were significantly affected by sowing date, specially, seed yield per faddan. The results revealed significant differences among the eight faba bean genotypes in their performance in both of number of days to flowering and maturity. The earliest faba bean genotypes were Giza-843, Sakha-3 and Giza-716 in both Sids and Mallawy. It was found that, late sowing showed a shorter period until to maturity. Genotypes produced higher seed yield/plant at the $2^{\text {nd }}$ sowing date (November $1^{\text {st }}$ ). Genotypes produced heaviest 100 -seeds in the $1^{\text {st }}$ sowing date, because, plants stayed longer to reach maturity, relative to those planted in both of Novamber $1^{\text {st }}$ or November $15^{\text {th }}$, since seeds given much time to grow. Sakha-3 in both Mallawy and Sids, attained the highest seed yield (ton/fad.). The second sowing date (November $1^{\text {st }}$ ) was most proper and any of Sakha-1 and Sakha-3 Sids-19 might be adopted to Middle Egypt.
\end{abstract}

Keywords: faba bean, sowing dates, yield components, upper Egypt.

\section{INTRODUCTION}

Faba bean is the most important food legume crop in Egypt, as a source of plant protein, and plays a good role in farming, as a break crop in intensive cereal cropping systems. Breeding activities have been employed for combining genes for adaptability and high yield from elite faba bean genotypes with those for earliness (Bekheit, 2007 and Mohamed, 2010). Moreover, in Egypt, there were success in breeding efforts for faba bean cultivars combining both earliness and high yield in one genotype, such as Giza 716, Sakha 1 and others. During the last five years (2015-2019), the cultivated area, in Egypt, was about 113.810 faddans, with an average productivity of 9.2 ardabs/ faddan.

Sowing date is an important factor which significantly affects the timing and duration of vegetative and reproductive stages consequently yield and its components and seed quality. Many farmers intend to sow faba bean at the beginning of October, while, the optimum sowing date, for the commercial cultivars is recommended to be at mid- November, as reported by Refay (2001), ElDeeb et al. (2006), Hussein et al. (2006 )in upper Egypt, Amer et al (2008)in North delta and ElGalaly et al. (2008). Talal and Ghalib (2006) reported that, early planting resulted in a significant yield advantage (157\%), over the late sowing date. They concluded that, much of this advantage resulted from the extended period of vegetative growth which resulted in the improvement of several agronomic characters. Badran et al. (2010) indicated that, sowing date had a significant effect on number of days to maturity, number of branches/plant, number of seeds/plant, seed weight/plant, 100-seed weight and seed yield/faddan. Also, they concluded that, planting faba bean on Nov.15 ${ }^{\text {th }}$ improved growth characters; seed yield and its components of faba bean. Similarly, El-Metwally et al. (2013) showed that, sowing date faba bean at $25^{\text {th }}$ October recorded the highest values of growth characters and pigment content (total chlorophyll). While, the greatest values of yield and its components were resulted from sowing at $25^{\text {th }}$ November. Also Amer et al. (2008) and Badr et al. (2013) found that early sowing date produced the highest faba bean seed yield and its components.

The objective of this recent study was to detect the most convenient time of sowing for faba bean genotypes with promising levels of yield and earliness in Middle Egypt.

\section{MATERIALS AND METHODS}

Field experiments were conducted at Sids and Mallawy Research Stations, (Middle Egypt) during two successive seasons (2016/2017 and 2017/ 2018) to study the effect of sowing dates on the productivity of eight faba bean genotypes i.e.; Sakha-1, Sakha-3, Giza-843, Giza-716, Pop.-10, SB-1, BF-10 and Sids 19. The origin and pedigree of the studied genotypes were presented in Table 1. The chemical and physical soil properties of experimental soil at Sids and Mallawy agricultural research stations are recorded in Table (2). 
Experiments were carried- out in a split plot design. Sowing dates (Octobar $15^{\text {th }}$, November $1^{\text {st }}$ and November $15^{\text {th }}$ ) occupied the main plots. Whereas, faba bean genotypes were arranged in sub-plots. Data of the two years were combined when the assumption of error homogeneity can not be rejected (Barttlet,1937). The year $\mathrm{x}$ treatments was not significant, so that, means over years were presented.

Each plot involved five ridges each of threemeter long and 60 centimeters apart $\left(9 \mathrm{~m}^{2}\right)$. Days to $50 \%$ flowering and $90 \%$ maturity recorded. were recorded for each plot. At harvest, ten plants were taken randomly from each plot, were, the following characters were recorded: -
- Number of branches/plant

- Number of pods/plant

- Number of seeds/plant

- Seed weight/plant

- 100 seed weight $(\mathrm{g})$.

- Seed yield (ton/faddan.) was the five determined from ridges of each plot.

\section{Statistical analysis:-}

Data were statistically analyzed according to Sendecor and Cochran (1981). Means were compared by the L.S.D. value at 0.05 level of probability.

Table 1: The origin and pedigree of the studied faba bean genotypes.

\begin{tabular}{lll}
\hline Genotype & Origin & Pedigree \\
\hline Sakha-1 & Egypt & $716 / 724 / 88 / 620 / 283 / 85$ \\
Sakha-3 & Egypt & Single plant selection from Giza716 \\
Giza-843 & Egypt & $461 / 845 / 83 / 561 / 2076 / 85$ \\
Giza-716 & Egypt & $461 / 842 / 83 / 503 / 453 / 83$ \\
Population-10 & Egypt & Single seed-disent from Misr3 \\
SB-1 & Egypt & IcarusX Giza843 \\
BF-10 & Egypt & Co8/Fam/151 Ter6 X B7/9042/06 \\
Sids 19 & Egypt & (Giza 40 X Misr 2) X Giza 716 X T.W.) \\
\hline
\end{tabular}

Table 2: Chemical and physical soil properties of experimental soils at Sids and Mallawy research stations

\begin{tabular}{|c|c|c|}
\hline Properties & Sids & Mallawy \\
\hline \multicolumn{3}{|l|}{ Mechanical: } \\
\hline Sandy $\%$ & 9.50 & 7.90 \\
\hline Silt $\%$ & 31.9 & 54.50 \\
\hline Clay \% & 60.6 & 37.60 \\
\hline Textural grade & Clay & Silty Clay loam \\
\hline \multicolumn{3}{|l|}{ Chemical: } \\
\hline $\mathrm{pH}$ & 7.72 & 8.20 \\
\hline E.C. $(\mathrm{ds} / \mathrm{m})$ & 1.04 & 1.35 \\
\hline Organic matter $\%$ & 0.91 & 1.18 \\
\hline \multicolumn{3}{|l|}{ Soluble cations } \\
\hline $\mathrm{Ca}^{++}$ & 3.0 & 6.25 \\
\hline $\mathrm{Mg}^{+}$ & 1.36 & 0.76 \\
\hline $\mathrm{K}^{+}$ & 0.98 & 0.20 \\
\hline $\mathrm{Na}^{+}$ & 5.12 & 2.85 \\
\hline \multicolumn{3}{|l|}{ Soluble anions } \\
\hline $\mathrm{Co}_{3}^{-}$ & 0.00 & 0.00 \\
\hline $\mathrm{HcO}_{3}$ & 1.51 & 2.05 \\
\hline $\mathrm{Cl}^{-}$ & 1.72 & 2.25 \\
\hline $\mathrm{SO}_{4}^{-}$ & 7.23 & 5.85 \\
\hline Available N (ppm) & 62.46 & 20.35 \\
\hline Available P (ppm) & 7.62 & 8.15 \\
\hline Available K (ppm) & 311.60 & 183.0 \\
\hline
\end{tabular}




\section{RESULTS AND DISCUSSION}

\section{Effect of sowing date:}

Results of the combined analysis for the two experimental seasons 2016/2017 and 2017/2018 are presented for each location in Tables (3,4,5 and 6). As for the effect of sowing date, the results showed significant differences among the three sowing dates. The second sowing date (November $1^{\text {st }}$ )gave the highest values in number of branches, number of pods, number of seeds/plant, seed weight/plant $(\mathrm{g})$ and seed yield (ton/fad.), (4.8, 3.7), (20.6, 26.6), $(57.7,73.9),(53.8,67.6),(1.821,2.133)$ at Mallawy and Sids locations in the two growing seasons, respectively, On the other hand, the first sowing date (October $15^{\text {th }}$ ) gave the lowest values in number of branches, number of pods, number of seeds/plant, seed weight/plant (g) and seed yield (ton/fad.), at Mallawy and Sids locations, respectively, $(3.2,2.7)$, (15.2, 12.2), (34.6, 28.8), (28.0, 24.0), (1.023, 1.098). Badr et al. (2013) found that, sowing faba bean, on Oct $31^{\text {st }}$ produced, the highest number of pods, seeds/plant, seed yield/plant (g) and seed yield (ton/fad.). In addition, delaying sowing date, (Nov. $\left.15^{\text {th}}\right)$, significantly decreased, days to flowering and maturity, number of branches/ plant, number of pods/plant, number of seeds/plant, seed weight/plant (g), 100- seed weight (g) and seed yield (ton/fad.) (52.0, 53.5), (129.6, 124.8), (4.1, 3.4), (17.6, 18.3), (46.9, 41.6), (40.7, 36.0), (80.6, 75.3), (1.445, 1.697) in Mallawy and Sids locations respectively. The high 100 -seed weight $(\mathrm{g})$ obtained from early sowing, might due to the fact that plants had a sufficient longer vegetative period and better utilization of water and nutrients. These results coincided with those obtained by Abbas et al. (2010), Khalil et al. (2011), El -Metwally et al, (2013). and Badr et al. (2013).

\section{Effect of faba bean genotypes:}

Data recorded in Tables (3,4,5 and 6) showed that, the differences among faba bean genotypes were significant for days to flowering and maturity, number of branches, number of pods, number of seeds/plant, seed yield/plant, 100- seed weight and seed yield/fad., in Mallawy and Sids locations. Sakha 3 genotype was the earliest (50.8 day) followed by Giza 843 genotype (54.2 day) in Mallawy. Moreover, Sakha1 genotype was the earliest (53.9 day) followed by SB-1 genotype (54.0 day) in Sids. On the other hand, Sakha 3 genotype, gave the highest values in number of branches, number of pods, number of seeds/plant, seed weight/plant, 100-seed weight and seed yield/fad., (5.1, 3.7), (21.7, 23.9), (56.5, 61.0), $(52.8,55.6),(92.8,86.0)$ and $(1.838,1.813)$ in Mallawy and Sids locations, respectively. Moreover, the least values for these characters were recorded by SB-1 and BF-10 genotypes in Mallawy and Pop- 10 and BF-10 gentypes in Sids location. These results were, in general, agreement with those of Mekky et al. (2003), Abbas et al., (2010) and Badr et al. (2013) and Abido and Seadh (2014), Mohamed (2012) who concluded that, there were significant diffierences among genotypes in most traits under sowing dates.

\section{Effect of interaction:-}

Data of Tables (3, 4, 5 and 6) revealed that, the interaction effect between faba bean genotypes and sowing dates was significant in days to flowering and maturity, number of branches, number of pods, number of seeds/plant, seed weight/plant and seed yield/fad., in Mallawy. Also, the interaction between sowing date and faba bean genotypes was significant for days to flowering and maturity, number of branches, number of pods, number of seeds, seed weight/plant (g), seed yield (ton/fad.), 100-seed weight (g) at Sids.

It was clear from the data at Sakha3 and Sids 19 genotypes on Nov. $1^{\text {st }}$ sowing date gave the highest values for seed yield/fad. in Mallawy and Sids Locations.

\section{CONCLUSION}

Generally, it can be concluded that, sowing faba bean genotypes on Nov. $1^{\text {st }}$ might improve the growth and yield. Sakha 3 cultivar and Sids 19 genotype were preferably recommended because of its superior response to such conditions. 
Table 3: Days to flowering, maturity, number of branches/plant and number of pods/plant of eight faba bean genotypes as affected by sowing dates at Mallawy research station (Combined over the two seasons 2016/2017 and 2017-2018)

\begin{tabular}{|c|c|c|c|c|c|c|c|c|c|c|c|c|c|}
\hline \multirow{3}{*}{ Variable } & & \multicolumn{12}{|c|}{ Mallawy location } \\
\hline & & \multicolumn{3}{|c|}{ 50\% Flowering } & \multicolumn{3}{|c|}{ 90\% Maturity } & \multicolumn{3}{|c|}{$\begin{array}{c}\text { No. of } \\
\text { branches/plant }\end{array}$} & \multicolumn{3}{|c|}{ No. of pods/plant } \\
\hline & & Y1 & $\mathbf{Y 2}$ & Mean & Y1 & Y2 & Mean & Y1 & Y2 & Mean & Y1 & Y2 & Mean \\
\hline \multirow{3}{*}{$\begin{array}{l}\text { Sowing } \\
\text { date(S) }\end{array}$} & Oct15 $^{\text {th }}$ & 58.4 & 60.6 & 59.5 & 135.6 & 136.6 & 136.1 & 3.3 & 3.0 & 3.2 & 15.7 & 14.7 & 15.2 \\
\hline & Nov. $1^{\text {st }}$ & 54.9 & 57.4 & 56.1 & 133.8 & 133.0 & 133.4 & 5.2 & 4.4 & 4.8 & 21.4 & 19.8 & 20.6 \\
\hline & Nov. $15^{\text {th }}$ & 51.2 & 52.9 & 52.0 & 131.9 & 127.4 & 129.6 & 4.5 & 3.7 & 4.1 & 15.5 & 16.8 & 17.6 \\
\hline L.S.D. 0.05 & & 2.0 & 2.1 & 4.7 & 2.3 & 2.6 & 4.8 & 0.4 & 0.7 & 0.3 & 1.0 & 1.6 & 0.8 \\
\hline \multirow{8}{*}{$\begin{array}{c}\text { Genotypes } \\
\text { (G) }\end{array}$} & Sakha-1 & 55.1 & 56.6 & 55.8 & 132.2 & 131.3 & 131.7 & 5.0 & 4.1 & 4.5 & 20.3 & 18.7 & 19.5 \\
\hline & Sakha-3 & 49.3 & 52.3 & 50.8 & 130.8 & 130.1 & 130.5 & 5.4 & 4.8 & 5.1 & 22.8 & 20.5 & 21.7 \\
\hline & Giza 843 & 52.6 & 55.8 & 54.2 & 130.2 & 129.7 & 130.0 & 4.6 & 4.0 & 4.3 & 18.2 & 17.7 & 18.0 \\
\hline & Giza-716 & 54.6 & 57.8 & 56.2 & 132.4 & 131.0 & 131.7 & 3.9 & 3.4 & 3.6 & 17.3 & 15.9 & 16.6 \\
\hline & Pop.10 & 59.2 & 60.4 & 59.8 & 136.7 & 134.4 & 135.6 & 4.3 & 3.3 & 3.8 & 17.7 & 15.9 & 16.8 \\
\hline & SB-1 & 57.4 & 58.8 & 58.1 & 138.1 & 134.5 & 136.3 & 3.4 & 3.1 & 3.2 & 16.4 & 15.0 & 15.7 \\
\hline & BF-10 & 55.4 & 56.5 & 56.0 & 134.3 & 134.0 & 134.1 & 3.5 & 3.1 & 3.3 & 16.2 & 14.8 & 15.5 \\
\hline & Sids-19 & 55.1 & 57.4 & 56.2 & 135.5 & 133.6 & 134.6 & 4.8 & 4.1 & 4.4 & 19.5 & 18.2 & 18.9 \\
\hline L.S.D. 0.05 & & 1.9 & 1.9 & 5.2 & 2.9 & 3.1 & 3.9 & 0.4 & 0.3 & 0.4 & 1.1 & 1.2 & 0.7 \\
\hline \multirow{8}{*}{$\begin{array}{l}\text { Oct } 15^{\text {th }} \\
\text { (S1) }\end{array}$} & Sakha-1 & 57.6 & 59.6 & 58.6 & 136.0 & 136.7 & 136.3 & 3.8 & 3.4 & 3.6 & 16.3 & 16.8 & 16.5 \\
\hline & Sakha-3 & 51.6 & 56.3 & 54.0 & 132.2 & 134.0 & 133.1 & 3.9 & 3.5 & 3.7 & 19.7 & 17.3 & 18.5 \\
\hline & Giza 843 & 55.6 & 59.3 & 57.5 & 131.6 & 132.6 & 132.1 & 3.4 & 3.1 & 3.3 & 15.7 & 14.5 & 15.1 \\
\hline & Giza-716 & 59.0 & 62.0 & 60.5 & 134.6 & 135.6 & 135.1 & 3.1 & 2.8 & 2.9 & 15.0 & 13.9 & 14.4 \\
\hline & Pop.10 & 63.9 & 64.0 & 63.8 & 139.0 & 139.0 & 139.0 & 3.3 & 3.1 & 3.2 & 15.3 & 14.3 & 14.8 \\
\hline & SB-1 & 61.3 & 62.6 & 62.0 & 139.6 & 139.6 & 139.6 & 3.0 & 2.7 & 2.9 & 14.2 & 13.3 & 13.7 \\
\hline & BF-10 & 59.6 & 60.0 & 59.8 & 135.0 & 138.6 & 136.8 & 2.8 & 2.7 & 2.7 & 13.7 & 12.4 & 13.1 \\
\hline & Sids-19 & 59.0 & 61.3 & 60.1 & 136.6 & 137.0 & 136.8 & 3.5 & 3.1 & 3.3 & 16.1 & 15.2 & 15.7 \\
\hline \multirow{8}{*}{$\begin{array}{l}\text { Nov. } 1^{\text {st }} \\
\text { (S2) }\end{array}$} & Sakha-1 & 55.3 & 57.3 & 56.3 & 132.6 & 131.3 & 132.0 & 5.6 & 4.5 & 5.1 & 23.3 & 20.0 & 21.6 \\
\hline & Sakha-3 & 49.6 & 52.0 & 50.8 & 131.6 & 131.0 & 131.3 & 6.7 & 6.0 & 6.3 & 25.5 & 23.1 & 24.6 \\
\hline & Giza843 & 52.0 & 56.0 & 54.0 & 130.0 & 130.0 & 130.1 & 5.6 & 4.7 & 5.1 & 20.6 & 21.3 & 20.9 \\
\hline & Giza-716 & 55.3 & 58.3 & 56.8 & 132.0 & 131.6 & 131.8 & 4.1 & 4.1 & 4.1 & 19.3 & 18.5 & 18.9 \\
\hline & Pop.10 & 58.3 & 60.0 & 59.1 & 136.6 & 135.0 & 135.8 & 5.2 & 3.8 & 4.5 & 20.4 & 18.3 & 19.4 \\
\hline & SB-1 & 57.3 & 59.3 & 58.3 & 138.0 & 135.3 & 136.6 & 3.6 & 3.5 & 3.5 & 18.6 & 16.4 & 17.5 \\
\hline & BF-10 & 56.0 & 58.0 & 57.0 & 134.3 & 135.3 & 134.8 & 4.4 & 3.5 & 4.0 & 19.2 & 18.0 & 18.6 \\
\hline & Sids- 19 & 55.7 & 58.3 & 57.0 & 135.6 & 134.0 & 134.8 & 6.4 & 5.0 & 5.7 & 24.8 & 23.0 & 23.9 \\
\hline \multirow{8}{*}{$\begin{array}{l}\text { Nov.15 } \\
\text { (S3) }\end{array}$} & Sakha-1 & 52.3 & 53.0 & 52.6 & 128.0 & 126.0 & 127.0 & 5.6 & 4.4 & 5.0 & 21.4 & 19.4 & 20.4 \\
\hline & Sakha-3 & 46.6 & 48.6 & 47.6 & 128.6 & 125.3 & 127.0 & 5.7 & 5.0 & 5.3 & 23.3 & 21.1 & 22.2 \\
\hline & Giza843 & 50.3 & 52.3 & 51.3 & 129.0 & 126.3 & 127.6 & 4.8 & 4.1 & 4.4 & 18.4 & 17.4 & 17.9 \\
\hline & Giza-716 & 49.6 & 53.3 & 51.5 & 130.6 & 125.6 & 128.1 & 4.4 & 3.3 & 3.8 & 17.7 & 15.3 & 16.5 \\
\hline & Pop.10 & 55.6 & 57.3 & 56.5 & 134.6 & 129.3 & 132.0 & 4.3 & 3.1 & 3.7 & 17.4 & 15.2 & 16.3 \\
\hline & SB-1 & 53.6 & 54.6 & 54.1 & 136.6 & 128.6 & 132.6 & 3.6 & 3.1 & 3.3 & 16.6 & 15.2 & 15.9 \\
\hline & BF-10 & 50.6 & 51.6 & 51.1 & 133.6 & 128.0 & 130.8 & 3.4 & 3.0 & 3.2 & 15.8 & 14.0 & 14.9 \\
\hline & Sids 19 & 50.6 & 52.6 & 51.6 & 134.3 & 130.0 & 132.1 & 4.4 & 4.1 & 4.3 & 17.8 & 16.5 & 17.1 \\
\hline L.S.D. 0.05 & & 1.2 & 1.3 & 1.3 & 1.9 & 1.9 & 1.3 & 0.8 & 0.6 & 0.5 & 1.8 & 2.0 & 1.9 \\
\hline
\end{tabular}

Y1- the first season 2016/2017, Y2- the second season 2017/2018. 
Alex. J. Agric. Sci.

Table 4: Number of seeds/plant, seed weight/plant (g), 100-seed weight (g) and seed yield (ton/fad.) of eight faba bean genotypes as affected by sowing dates at Mallawy research station (Combined over the two seasons 2016/2017 and 2017-2018)

\begin{tabular}{|c|c|c|c|c|c|c|c|c|c|c|c|c|c|}
\hline \multirow{3}{*}{ Variable } & & \multicolumn{12}{|c|}{ Mallawy location } \\
\hline & & \multicolumn{3}{|c|}{ No. of seeds/plant } & \multicolumn{3}{|c|}{ Seed weight/plant (g) } & \multicolumn{3}{|c|}{ 100-seed weight $(\mathrm{g})$} & \multicolumn{3}{|c|}{ Seed yield (ton/fed.) } \\
\hline & & Y1 & Y2 & Mean & Y1 & Y2 & Mean & Y1 & Y2 & Mean & Y1 & Y2 & Mean \\
\hline \multirow{3}{*}{$\begin{array}{l}\text { Sowing } \\
\text { date(S) }\end{array}$} & Oct15 ${ }^{\text {th }}$ & 32.0 & 37.2 & 34.6 & 26.2 & 29.8 & 28.0 & 92.9 & 92.5 & 92.7 & 1.069 & 0.977 & 1.023 \\
\hline & Nov. $1^{\text {st }}$ & 58.9 & 56.5 & 57.7 & 55.1 & 52.5 & 53.8 & 85.5 & 86.5 & 86.2 & 1.883 & 1.759 & 1.821 \\
\hline & Nov. $15^{\text {th }}$ & 47.3 & 46.5 & 46.9 & 40.9 & 40.5 & 40.7 & 81.6 & 79.6 & 80.6 & 1.532 & 1.359 & 1.445 \\
\hline L.S.D. 0.05 & & 2.9 & 4.1 & & 2.1 & 1.8 & 3.8 & 1.7 & 1.5 & 2.2 & 0.96 & 0.165 & 0.99 \\
\hline \multirow{8}{*}{$\begin{array}{c}\text { Genotypes } \\
\text { (G) }\end{array}$} & Sakha-1 & 50.7 & 49.8 & 50.3 & 47.0 & 45.0 & 46.0 & 91.5 & 89.6 & 90.6 & 1.738 & 1.569 & 1.653 \\
\hline & Sakha-3 & 55.8 & 57.2 & 56.5 & 52.8 & 52.7 & 52.8 & 94.2 & 91.4 & 92.8 & 1.896 & 1.781 & 1.838 \\
\hline & Giza 843 & 46.4 & 49.7 & 48.0 & 42.1 & 44.3 & 43.2 & 89.9 & 87.9 & 88.5 & 1.645 & 1.503 & 1.573 \\
\hline & Giza-716 & 42.3 & 43.3 & 42.8 & 36.4 & 37.8 & 37.1 & 85.1 & 85.9 & 85.5 & 1.328 & 1.262 & 1.294 \\
\hline & Pop.10 & 44.2 & 41.9 & 43.0 & 38.5 & 35.9 & 37.2 & 86.2 & 84.7 & 85.5 & 1.350 & 1.171 & 1.260 \\
\hline & SB-1 & 39.0 & 41.2 & 40.1 & 30.9 & 33.8 & 32.3 & 78.4 & 80.9 & 79.7 & 1.129 & 1.033 & 1.081 \\
\hline & BF-10 & 39.0 & 39.2 & 39.1 & 31.0 & 31.9 & 31.5 & 77.8 & 80.2 & 79.0 & 1.129 & 1.017 & 1.073 \\
\hline & Sids-19 & 51.0 & 51.4 & 51.2 & 47.2 & 46.3 & 46.7 & 91.2 & 88.7 & 89.9 & 1.743 & 1.584 & 1.663 \\
\hline L.S.D. 0.05 & & 2.4 & 2.5 & 1.8 & 5.1 & 3.1 & 2.9 & 5.2 & 3.5 & 4.4 & 0.112 & 0.96 & 0.106 \\
\hline \multirow{8}{*}{$\begin{array}{l}\text { Oct } 15^{\text {th }} \\
\text { (S1) }\end{array}$} & Sakha-1 & 34.6 & 41.2 & 37.9 & 30.1 & 34.6 & 32.3 & 96.2 & 94.2 & 95.2 & 1.369 & 1.204 & 1.286 \\
\hline & Sakha-3 & 39.6 & 45.0 & 42.3 & 35.6 & 38.7 & 37.1 & 98.0 & 96.7 & 97.4 & 1.384 & 1.343 & 1.363 \\
\hline & Giza 843 & 31.0 & 40.0 & 35.5 & 26.3 & 32.2 & 29.2 & 94.2 & 95.4 & 94.8 & 1.194 & 1.038 & 1.116 \\
\hline & Giza-716 & 30.0 & 33.5 & 21.7 & 24.1 & 26.2 & 25.1 & 90.2 & 93.6 & 91.9 & 0.863 & 0.816 & 0.939 \\
\hline & Pop.10 & 30.9 & 34.0 & 32.4 & 24.9 & 27.1 & 26.0 & 93.8 & 91.3 & 92.5 & 0.961 & 0.836 & 0.898 \\
\hline & SB-1 & 28.4 & 33.2 & 30.8 & 20.8 & 25.4 & 23.1 & 85.2 & 84.2 & 84.7 & 0.800 & 0.728 & 0.764 \\
\hline & BF-10 & 27.0 & 30.0 & 28.5 & 19.0 & 21.6 & 20.3 & 88.1 & 88.1 & 88.1 & 0.790 & 0.655 & 0.723 \\
\hline & Sids-19 & 34.2 & 40.5 & 37.3 & 29.5 & 33.0 & 31.2 & 97.7 & 96.4 & 97.0 & 1.193 & 1.198 & 1.195 \\
\hline \multirow{8}{*}{$\begin{array}{l}\text { Nov. } 1^{\text {st }} \\
\text { (S2) }\end{array}$} & Sakha-1 & 63.4 & 57.2 & 60.3 & 61.2 & 53.7 & 57.4 & 91.5 & 91.2 & 91.4 & 2.015 & 1.880 & 1.974 \\
\hline & Sakha-3 & 71.8 & 68.0 & 69.9 & 70.0 & 65.3 & 67.6 & 94.7 & 92.2 & 93.5 & 2.367 & 2.242 & 2.304 \\
\hline & Giza843 & 58.0 & 60.0 & 59.0 & 54.6 & 57.4 & 56.0 & 90.7 & 88.2 & 89.5 & 1.943 & 1.978 & 1.960 \\
\hline & Giza-716 & 52.4 & 53.2 & 52.7 & 47.5 & 49.9 & 48.7 & 84.9 & 85.5 & 85.3 & 1.689 & 1.632 & 1.660 \\
\hline & Pop.10 & 57.6 & 49.1 & 53.3 & 54.1 & 44.9 & 49.5 & 83.2 & 83.5 & 83.4 & 1.741 & 1.482 & 1.611 \\
\hline & SB-1 & 46.0 & 48.1 & 47.0 & 39.3 & 40.9 & 40.1 & 76.9 & 82.6 & 79.7 & 1.483 & 1.332 & 1.407 \\
\hline & BF-10 & 52.3 & 50.9 & 51.6 & 46.1 & 44.9 & 45.5 & 75.2 & 80.4 & 77.8 & 1.570 & 1.436 & 1.503 \\
\hline & Sids-19 & 69.8 & 65.2 & 67.5 & 68.3 & 63.2 & 65.7 & 89.3 & 88.2 & 88.8 & 2.258 & 2.087 & 2.173 \\
\hline \multirow{8}{*}{$\begin{array}{l}\text { Nov. } 15^{\text {th }} \\
\text { (S3) }\end{array}$} & Sakha-1 & 54.3 & 51.0 & 52.6 & 49.7 & 46.7 & 48.2 & 86.8 & 83.6 & 85.2 & 1.829 & 1.622 & 1.725 \\
\hline & Sakha-3 & 55.9 & 58.7 & 57.3 & 52.9 & 54.2 & 53.5 & 89.5 & $85 . .9$ & 87.7 & 1.937 & 1.756 & 1.846 \\
\hline & Giza843 & 50.3 & 49.0 & 49.6 & 45.6 & 43.3 & 44.4 & 84.7 & 80.2 & 82.5 & 1.798 & 1.492 & 1.645 \\
\hline & Giza-716 & 44.7 & 43.4 & 44.0 & 37.6 & 37.3 & 37.4 & 80.3 & 78.1 & 79.2 & 1.431 & 1.338 & 1.384 \\
\hline & Pop.10 & 44.0 & 42.8 & 43.4 & 36.5 & 35.9 & 36.2 & 81.6 & 79.5 & 80.5 & 1.348 & 1.193 & 1.270 \\
\hline & SB-1 & 42.7 & 42.4 & 42.5 & 32.8 & 35.1 & 33.9 & 73.2 & 76.0 & 74.6 & 1.105 & 1.038 & 1.071 \\
\hline & BF-10 & 37.6 & 36.6 & 37.1 & 28.1 & 29.3 & 28.7 & 70.0 & 72.0 & 71.0 & 1.028 & 0.960 & 0.994 \\
\hline & Sids-19 & 49.2 & 48.6 & 48.9 & 43.9 & 42.8 & 43.3 & 86.7 & 81.4 & 84.1 & 1.777 & 1.467 & 1.622 \\
\hline L.S.D. 0.05 & & 4.2 & 4.4 & 4.5 & N.S & 5.5 & 6.4 & N.S & N.S & 0.195 & N.S & 0.166 & 0.136 \\
\hline
\end{tabular}

Y1- the first season 2016/2017, Y2- the second season 2017/2018. 
Table 5: Days to flowering, maturity, number of branches/plant and number of pods/plant of eight faba bean genotypes as affected by sowing dates at Sids research station (Combined over the two seasons 2016/2017 and 2017-2018)

\begin{tabular}{|c|c|c|c|c|c|c|c|c|c|c|c|c|c|}
\hline \multirow{3}{*}{ Variable } & & \multicolumn{12}{|c|}{ Sids location } \\
\hline & & \multicolumn{3}{|c|}{$50 \%$ Flowering } & \multicolumn{3}{|c|}{$90 \%$ Maturity } & \multicolumn{3}{|c|}{$\begin{array}{c}\text { No. of } \\
\text { branches/plant }\end{array}$} & \multicolumn{3}{|c|}{ No. of pods/plant } \\
\hline & & Y1 & Y2 & Mean & Y1 & Y2 & Mean & Y1 & Y2 & Mean & Y1 & Y2 & Mean \\
\hline \multirow{3}{*}{$\begin{array}{l}\text { Sowing } \\
\text { date(S) }\end{array}$} & Oct15 & 61.5 & 63.0 & 62.2 & 140.9 & 139.5 & 140.9 & 2.9 & 2.5 & 2.7 & 12.7 & 11.8 & 12.2 \\
\hline & Nov. $1^{\text {st }}$ & 58.7 & 60.0 & 59.3 & 136.4 & 134.8 & 136.4 & 3.8 & 3.6 & 3.7 & 27.9 & 25.3 & 26.6 \\
\hline & Nov. $15^{\text {th }}$ & 53.0 & 54.0 & 53.5 & 126.2 & 123.4 & 126.2 & 3.5 & 3.2 & 3.4 & 18.4 & 18.2 & 18.3 \\
\hline \multicolumn{2}{|l|}{ L.S.D. 0.05} & 3.0 & 3.8 & 2.0 & 1.5 & 1.9 & 1.6 & 0.5 & 0.6 & 0.4 & 1 & 1.6 & 0.8 \\
\hline \multirow{8}{*}{$\begin{array}{c}\text { Genotypes } \\
\text { (G) }\end{array}$} & Sakha-1 & 52.8 & 54.9 & 53.9 & 132.3 & 131.2 & 131.7 & 3.4 & 3.2 & 3.3 & 19.6 & 18.5 & 19.0 \\
\hline & Sakha-3 & 58.3 & 58.5 & 58.4 & 135.4 & 134.1 & 134.8 & 3.9 & 3.6 & 3.7 & 24.7 & 23.2 & 23.9 \\
\hline & Giza 843 & 58.6 & 58.8 & 58.7 & 134.3 & 133.0 & 133.6 & 3.3 & 2.9 & 3.1 & 16.8 & 15.9 & 16.3 \\
\hline & Giza-716 & 61.4 & 63.4 & 62.4 & 131.4 & 129.0 & 130.2 & 3.4 & 3.1 & 3.2 & 18.3 & 16.8 & 17.6 \\
\hline & Pop.10 & 55.3 & 56.4 & 55.8 & 135.0 & 133.2 & 134.1 & 3.1 & 2.8 & 2.9 & 16.5 & 15.3 & 15.9 \\
\hline & SB-1 & 52.3 & 55.8 & 54.0 & 138.0 & 135.2 & 136.6 & 3.4 & 3.1 & 3.3 & 21.1 & 20.3 & 20.7 \\
\hline & BF-10 & 60.8 & 60.5 & 60.7 & 134.3 & 132.5 & 133.4 & 3.3 & 3.0 & 3.2 & 18.0 & 17.0 & 17.5 \\
\hline & Sids-19 & 62.0 & 63.5 & 62.9 & 135.3 & 132.5 & 133.9 & 3.6 & 3.4 & 3.5 & 22.2 & 20.7 & 21.4 \\
\hline \multicolumn{2}{|l|}{ L.S.D. 0.05} & 1.9 & 2.5 & 1.7 & 1.8 & 2.6 & 1.3 & 0.2 & 0.2 & 0.3 & 2.3 & 3.9 & 1.5 \\
\hline \multirow{8}{*}{$\begin{array}{l}\text { Oct } 15^{\text {th }} \\
\text { (S1) }\end{array}$} & Sakha-1 & 55.0 & 57.3 & 56.1 & 138.6 & 137.0 & 137.8 & 3.2 & 3.0 & 3.1 & 13.2 & 13.5 & 13.3 \\
\hline & Sakha-3 & 62.3 & 62.3 & 62.3 & 142.3 & 141.2 & 141.7 & 3.1 & 2.8 & 2.9 & 13.0 & 12.8 & 12.9 \\
\hline & Giza 843 & 62.3 & 63.9 & 63.0 & 141.0 & 140.1 & 140.5 & 2.8 & 2.4 & 2.6 & 12.5 & 11.0 & 11.7 \\
\hline & Giza-716 & 64.6 & 68.3 & 66.5 & 137.6 & 136.0 & 136.8 & 3.0 & 2.6 & 2.8 & 12.9 & 11.3 & 12.1 \\
\hline & Pop.10 & 59.3 & 59.0 & 59.1 & 142.6 & 142.0 & 142.3 & 2.7 & 2.3 & 2.5 & 12.4 & 11.9 & 12.1 \\
\hline & SB-1 & 56.3 & 61.0 & 58.6 & 145.3 & 143.1 & 144.2 & 2.7 & 2.3 & 2.5 & 12.2 & 11.8 & 12.0 \\
\hline & BF-10 & 65.3 & 64.0 & 64.6 & 140.0 & 139.0 & 139.5 & 2.9 & 2.5 & 2.7 & 12.6 & 11.0 & 11.8 \\
\hline & Sids-19 & 66.6 & 68.3 & 67.5 & 139.6 & 138.1 & 138.8 & 3.0 & 2.8 & 2.9 & 12.9 & 11.5 & 12.2 \\
\hline \multirow{8}{*}{$\begin{array}{l}\text { Nov. } 1^{\text {st }} \\
\text { (S2) }\end{array}$} & Sakha-1 & 55.0 & 55.7 & 55.3 & 133.6 & 133.6 & 133.6 & 3.8 & 3.7 & 3.7 & 29.8 & 27.2 & 28.5 \\
\hline & Sakha-3 & 59.0 & 59.6 & 59.3 & 136.6 & 137.0 & 136.8 & 4.6 & 4.2 & 4.4 & 35.5 & 33.0 & 34.2 \\
\hline & Giza843 & 59.0 & 58.6 & 58.8 & 138.0 & 136.0 & 137.0 & 3.7 & 3.4 & 3.5 & 22.4 & 20.1 & 21.2 \\
\hline & Giza-716 & 63.0 & 64.5 & 63.7 & 134.3 & 131.5 & 132.9 & 3.6 & 3.1 & 3.3 & 22.7 & 19.8 & 21.3 \\
\hline & Pop.10 & 56.0 & 57.6 & 56.8 & 138.0 & 137.5 & 137.7 & 3.6 & 3.3 & 3.4 & 22.2 & 20.0 & 21.1 \\
\hline & SB-1 & 53.6 & 56.4 & 55.0 & 140.0 & 137.2 & 138.6 & 4.0 & 2.3 & 3.9 & 32.6 & 30.0 & 31.3 \\
\hline & BF-10 & 60.6 & 60.9 & 60.7 & 134.3 & 133.6 & 133.9 & 3.7 & 2.5 & 3.6 & 23.7 & 21.4 & 22.5 \\
\hline & Sids 19 & 63.3 & 66.4 & 64.8 & 136.3 & 132.1 & 134.2 & 4.1 & 2.8 & 4.0 & 34.5 & 31.5 & 33.0 \\
\hline \multirow{8}{*}{$\begin{array}{l}\text { Nov. } 15^{\text {th }} \\
\text { (S3) }\end{array}$} & Sakha-1 & 48.6 & 51.6 & 50.1 & 124.6 & 123.0 & 123.8 & 3.3 & 3.0 & 3.1 & 15.8 & 15.0 & 15.4 \\
\hline & Sakha-3 & 53.6 & 53.6 & 53.6 & 127.3 & 124.3 & 125.8 & 4.0 & 3.8 & 3.9 & 25.6 & 23.9 & 24.7 \\
\hline & Giza843 & 54.6 & 54.3 & 54.5 & 124.0 & 123.0 & 123.5 & 3.4 & 3.0 & 3.2 & 15.5 & 16.6 & 16.0 \\
\hline & Giza-716 & 56.6 & 57.3 & 57.0 & 122.3 & 119.5 & 120.9 & 3.8 & 3.6 & 3.7 & 19.5 & 19.3 & 19.4 \\
\hline & Pop.10 & 50.6 & 52.6 & 51.6 & 124.3 & 120.3 & 122.3 & 3.1 & 2.8 & 2.9 & 15.1 & 14.2 & 14.6 \\
\hline & SB-1 & 47.0 & 50.0 & 48.5 & 128.6 & 125.4 & 127.0 & 3.7 & 3.4 & 3.5 & 18.5 & 19.2 & 18.8 \\
\hline & BF-10 & 56.6 & 56.6 & 56.6 & 128.6 & 125.0 & 126.8 & 3.5 & 3.1 & 3.3 & 17.9 & 18.8 & 18.3 \\
\hline & Sids-19 & 56.0 & 56.0 & 56.0 & 130.0 & 127.3 & 128.6 & 3.7 & 3.6 & 3.6 & 19.3 & 19.1 & 19.2 \\
\hline L.S.D. 0.05 & & N.S & N.S & 1.7 & 2.1 & 2.4 & 3.5 & N.S & 0.4 & 3.6 & $\begin{array}{l}3.6 \\
\end{array}$ & 4.5 & 3.9 \\
\hline
\end{tabular}

Y1- the first season 2016/2017, Y2- the second season 2017/2018. 
Table 6: Number of seeds/plant, seed weight/plant (g), 100-seed weight (g) and seed yield (ton/fad.) of eight faba bean genotypes as affected by sowing dates at Sids research station (Combined over the two seasons 2016/2017 and 2017-2018)

\begin{tabular}{|c|c|c|c|c|c|c|c|c|c|c|c|c|c|}
\hline \multirow{3}{*}{ Variable } & & \multicolumn{12}{|c|}{ Sids location } \\
\hline & & \multicolumn{3}{|c|}{ No. of seeds/plant } & \multicolumn{3}{|c|}{ Seed weight/plant (g) } & \multicolumn{3}{|c|}{ 100-seed weight (g) } & \multicolumn{3}{|c|}{ Seed yield (ton/fed.) } \\
\hline & & Y1 & Y2 & Mean & Y1 & Y2 & Mean & Y1 & Y2 & Mean & Y1 & Y2 & Mean \\
\hline \multirow{3}{*}{$\begin{array}{l}\text { Sowing } \\
\text { date(S) }\end{array}$} & Oct15 ${ }^{\text {th }}$ & 35.2 & 22.4 & 28.8 & 25.1 & 23.0 & 24.0 & 89.4 & 91.4 & 90.4 & 1.181 & 1.015 & 1.098 \\
\hline & Nov. $1^{\text {st }}$ & 78.0 & 69.8 & 73.9 & 69.8 & 65.4 & 67.6 & 75.0 & 85.4 & 80.2 & 2.209 & 2.056 & 2.133 \\
\hline & Nov. $15^{\text {th }}$ & 44.4 & 38.9 & 41.6 & 33.5 & 38.4 & 36.0 & 70.8 & 79.8 & 75.3 & 1.761 & 1.634 & 1.697 \\
\hline L.S.D. 0.05 & & 5.2 & 7.2 & 4.3 & 1.8 & 3.8 & 2.4 & 1.5 & 2.2 & 3.4 & 0.92 & 0.161 & $\mathbf{0 . 4 3}$ \\
\hline \multirow{8}{*}{$\begin{array}{c}\text { Genotypes } \\
\text { (G) }\end{array}$} & Sakha-1 & 51.1 & 44.7 & 47.9 & 42.2 & 42.8 & 42.5 & 80.3 & 86.4 & 83.3 & 1.790 & 1.640 & 1.715 \\
\hline & Sakha-3 & 65.0 & 57.1 & 61.0 & 55.7 & 55.6 & 55.6 & 82.7 & 89.3 & 86.0 & 1.891 & 1.735 & 1.813 \\
\hline & Giza 843 & 46.3 & 36.5 & 41.4 & 36.5 & 35.0 & 35.7 & 76.0 & 84.5 & 80.2 & 1.686 & 1.533 & 1.601 \\
\hline & Giza-716 & 51.0 & 39.4 & 45.2 & 40.2 & 40.1 & 40.2 & 79.0 & 84.7 & 81.9 & 1.592 & 1.453 & 1.522 \\
\hline & Pop.10 & 44.0 & 33.3 & 38.6 & 34.3 & 31.8 & 33.0 & 73.8 & 83.0 & 78.4 & 1.542 & 1.380 & 1.461 \\
\hline & SB-1 & 55.5 & 45.9 & 50.7 & 45.6 & 44.0 & 44.8 & 77.6 & 84.3 & 81.0 & 1.680 & 1.537 & 1.608 \\
\hline & BF-10 & 48.3 & 41.3 & 44.8 & 38.7 & 39.3 & 39.0 & 77.5 & 84.8 & 81.1 & 1.707 & 1.575 & 1.641 \\
\hline & Sids-19 & 59.0 & 51.7 & 55.3 & 49.2 & 49.7 & 49.4 & 80.2 & 87.2 & 83.7 & 1.850 & 1.697 & 1.773 \\
\hline L.S.D. 0.05 & & 9.8 & 5.9 & 5.7 & 5.1 & 3.1 & 4.9 & 5.2 & 3.5 & 3.4 & 0.112 & 0.159 & 0.65 \\
\hline \multirow{8}{*}{$\begin{array}{l}\text { Oct } 15^{\text {th }} \\
\text { (S1) }\end{array}$} & Sakha-1 & 39.9 & 29.7 & 34.8 & 29.5 & 29.5 & 29.5 & 89.8 & 92.4 & 91.1 & 1.376 & 1.210 & 1.293 \\
\hline & Sakha-3 & 38.2 & 27.9 & 33.0 & 28.3 & 28.1 & 28.2 & 93.5 & 94.5 & 94.0 & 1.355 & 1.180 & 1.268 \\
\hline & Giza 843 & 34.0 & 17.1 & 25.5 & 23.5 & 18.6 & 21.0 & 88.9 & 90.2 & 89.5 & 1.101 & 0.941 & 1.021 \\
\hline & Giza-716 & 36.2 & 24.3 & 30.2 & 26.3 & 24.5 & 25.4 & 85.2 & 87.5 & 86.4 & 1.182 & 1.050 & 1.116 \\
\hline & Pop.10 & 31.7 & 16.9 & 24.3 & 21.5 & 18.1 & 19.8 & 87.7 & 88.9 & 88.3 & 1.059 & 0.803 & 0.930 \\
\hline & SB-1 & 30.0 & 16.7 & 23.3 & 19.8 & 17.4 & 18.6 & 90.1 & 93.3 & 91.7 & 0.918 & 0.781 & 0.849 \\
\hline & BF-10 & 35.1 & 20.1 & 27.6 & 24.9 & 21.0 & 22.9 & 89.0 & 90.7 & 89.8 & 1.127 & 1.021 & 1.074 \\
\hline & Sids-19 & 37.0 & 27.2 & 32.1 & 26.9 & 27.2 & 27.0 & 91.5 & 94.0 & 92.8 & 1.333 & 1.141 & 1.237 \\
\hline \multirow{8}{*}{$\begin{array}{l}\text { Nov. } 1^{\text {st }} \\
\quad(\mathrm{S} 2)\end{array}$} & Sakha-1 & 77.4 & 74.1 & 75.7 & 69.6 & 68.5 & 69.0 & 76.2 & 83.3 & 79.7 & 2.261 & 2.100 & 2.180 \\
\hline & Sakha-3 & 99.1 & 91.1 & 95.1 & 92.5 & 86.0 & 89.2 & 81.2 & 91.2 & 86.2 & 2.446 & 2.305 & 2.375 \\
\hline & Giza843 & 64.9 & 60.3 & 62.6 & 57.7 & 54.3 & 56.0 & 70.9 & 83.8 & 77.4 & 2.207 & 2.020 & 2.113 \\
\hline & Giza-716 & 60.8 & 45.8 & 53.2 & 50.2 & 49.1 & 49.6 & 79.6 & 86.5 & 83.1 & 1.751 & 1.610 & 1.680 \\
\hline & Pop.10 & 70.0 & 56.0 & 63.0 & 61.5 & 50.1 & 55.8 & 66.2 & 82.5 & 74.3 & 2.016 & 1.910 & 1.963 \\
\hline & SB-1 & 89.8 & 80.5 & 85.1 & 80.9 & 75.0 & 77.9 & 76.8 & 85.3 & 81.1 & 2.349 & 2.180 & 2.264 \\
\hline & BF-10 & 70.1 & 67.3 & 68.7 & 62.4 & 61.1 & 61.7 & 72.7 & 84.2 & 78.4 & 2.222 & 2.060 & 2.141 \\
\hline & Sids-19 & 91.6 & 83.9 & 87.7 & 83.8 & 79.1 & 81.4 & 76.2 & 86.0 & 81.1 & 2.423 & 2.270 & 2.346 \\
\hline \multirow{8}{*}{$\begin{array}{l}\text { Nov.15 } 15^{\text {th }} \\
\text { (S3) }\end{array}$} & Sakha-1 & 36.1 & 30.5 & 33.3 & 27.5 & 30.4 & 28.9 & 74.8 & 83.5 & 79.1 & 1.732 & 1.610 & 1.671 \\
\hline & Sakha-3 & 57.7 & 52.3 & 55.0 & 46.3 & 52.7 & 49.5 & 73.6 & 82.3 & 77.9 & 1.872 & 1.720 & 1.796 \\
\hline & Giza843 & 39.9 & 32.1 & 36.0 & 28.3 & 32.0 & 30.1 & 68.3 & 79.5 & 73.9 & 1.751 & 1.640 & 1.695 \\
\hline & Giza-716 & 55.9 & 48.3 & 52.1 & 44.2 & 46.8 & 45.2 & 72.3 & 80.1 & 76.2 & 1.843 & 1.700 & 1.771 \\
\hline & Pop.10 & 30.3 & 27.1 & 28.7 & 20.0 & 27.3 & 23.6 & 67.6 & 77.6 & 72.6 & 1.551 & 1.430 & 1.490 \\
\hline & SB-1 & 46.8 & 40.5 & 43.7 & 36.0 & 39.6 & 37.8 & 66.1 & 74.3 & 70.2 & 1.774 & 1.650 & 1.712 \\
\hline & BF-10 & 39.8 & 36.7 & 38.2 & 29.0 & 36.0 & 32.5 & 70.8 & 79.5 & 75.1 & 1.772 & 1.645 & 1.708 \\
\hline & Sids-19 & 48.4 & 44.0 & 46.2 & 36.9 & 42.8 & 39.9 & 73.0 & 81.5 & 77.2 & 1.793 & 1.680 & 1.736 \\
\hline L.S.D. 0.05 & & 10.0 & 11.4 & 14.2 & N.S & 5.4 & 11.9 & N.S & N.S & 8.7 & 0.112 & 0.119 & 0.170 \\
\hline
\end{tabular}

Y1- the first season 2016/2017, Y2- the second season 2017/2018. 


\section{REFERNCES}

Abbas, Z.,F. Sellami, M. Amri and M. Kharrat. 2010. Effect of sowing date on Orobanche foetida infection and seed yield of resistant and susceptible faba bean cultivars. Acta Phytopathol. Et entomol. Hungarica, 45(2): 267-275.

Abido, W.A. E.and S.E. Seadh. 2014. Rate of variations between field bean cultivars due to sowing dates and foliar sparing treatments. Sci. International 2 (1):1-12.

Amer, M.I., M.A. El-Borai and M.M. Radi. 2008. Response of three dates under different plant densities in North Delta. J. Agric.

Res. Tanta Univ.6 18(4): 591-599.

Badran,M.S.S. and M.Z.D.Ahmed. 2010. Effect of sowing dates and planting methods on growth characters, seed yield and its components of faba bean in Newly Reclaimed Lands. J. Agric.\& Env. Sci. 9 (1): 53- 66.

Badr, E.A.; M.W. Asal and A.A.Gehan. 2013. Effect of sowing dates and biofertilizer on growth attributes, yield and its components of two faba bean (Vicia faba, L.) cultivars. World Appl.Sci.J.28(4):494-498.

Bartlett, M.S. 1937. Statistical information and properties of sufficiency, Proc. Roy. Soc., London, Ser. A. 154: 124-137.

Bekheit, M. A. (2007). Breeding for earliness in faba bean (Vicia faba L.). Ph.D. Thesis, Fac. of Agric., Minia University, Egypt.

Cochran, W.G. and G.M. Cox. 1957. Experimental Designs.. John Wiley, New York, USA.

El-Deeb, M.A., A.H.A. Hussein, Kh.M. Yamani and T.S.A. El-Marsafawy. 2006. Response of new faba bean genotypes to different sowing dates and plant densities in New Valley. First Field Crops Conf. Proceedings. 20-22 Aug.Vol. 6: 358-362.

El-Galaly, O. A.M., M.I. Amer, S.A R.A.I. Abou-Moustafa and N.M.A. Mahmoud. 2008. Response of two faba bean (Vicia faba L.) promising lines and Sakha 3 cultivar to different sowing dates and plant densities. J. Agric. Res. 34(3): 647-661.
El-Metwally, I. M.; T. A. El-Shahawy and M.A. Ahmed. 2013. Effect of sowing dates and some broomrape control treatments on faba bean growth and yield J. Appi.Sci. Res. 9(1): 197-204.

Hussein, A.H., M.A. El-Deeb and Kh. ElYamani. 2006. Response of some new faba bean genotypes to different sowing dates and plant densities in the newly reclaimed lands in Upper Egypt. National Coordin. Meeting, Cairo, Egypt, 11-12 Sept., 2006.

Khalil, S.K.,A. Wahab and A.Z. Khan. 2011. Variation in leaf traits, yield and yield components of faba bean in response to planting dates and densities. Egypt, Acad. J. Biology Sci. 2(1): 35-43.

Mekky, M.S.; Z.R. Yehia and A.N.M. Nassar. 2003. Effect of sowing dates varieties and glyphosate application on broomrape (Orobanche crenata forsk.) and yield of faba bean (Vicia faba L.). Bull. Fac. Agric. Cairo University, 54 (4): 55-76.

Mohamed, A.K., 2010. Effect of sowing date, ridge direction, plant orientation and population on faba bean grain yield. fabis, 12: 11-12.

Mohamed, Marwa Kh. A. 2012. Effect of sowing date and broomrape control on productivity of some faba bean (Vicia faba,L) cultivars. M. Sc. Thesis, Fac. of Agric., Kafr-Elsheikh Unvi., Egypt.

Sendecor, G.W. and W.G. Cochran. 1981. Statistical methods. Seventh Edition. Iowa State Univ. Press, Ames, Iowa, USA.

Shad K. Khalil, Wahab A, Amanullah and Amir Zaman Khan. 2011. Variation in leaf traits, yield and yield components of faba bean in response to planting dates and densities. Egypt. Acad. J. biolog. Sci., 2(1): 35 - 43 (2011)

Refay YA. 2001. Effect of planting dates and plant density on two faba bean lines grown under the central region conditions of Saudi Arabia. Arab. Univ. J. Agric. Sci., 9: 79-93.

Talal, T. and S. Ghalib. 2006. Effect of planting date on faba bean (Vicia faba L.) nodulation and performance under semiarid conditions. World Journal of Agricultural Sciences, 2(4): 477-482. 


\section{تقييم بعض التراكيب الوراثية من الفول البلدي تحث ثلاث مواعيد زراعة

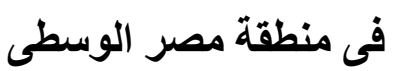 طارق صابر محمد، مروة خليل على محمد، رحاب احمد محمد عبدالرحمن} قسم بحوث المحاصيل البقولية معهد بحوث المحاصيل الحقلية، مركز البحوث الزر اعية- الجيزة، مصر

أجرى هذا البحث بمنطقة مصر الوسطى في كلا من المزرعة البحثية لمحطة البحوث الزراعية بملوي،

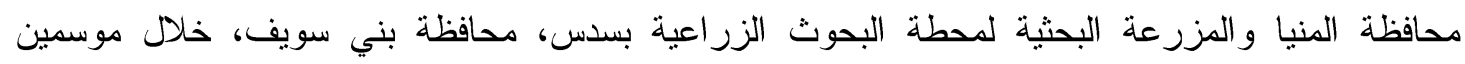

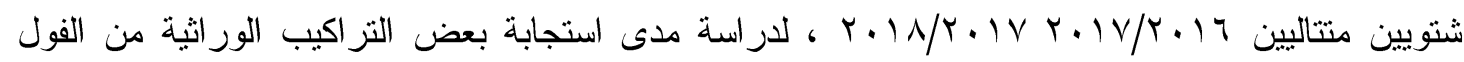

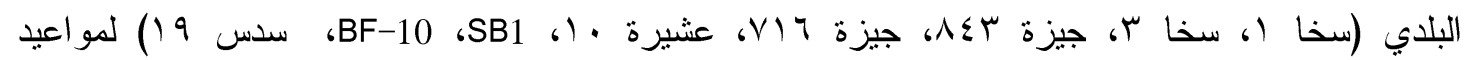

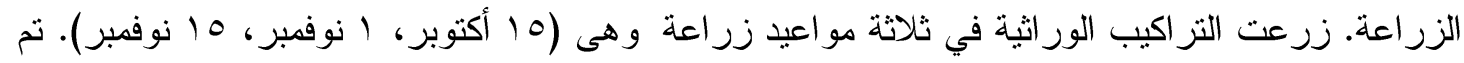

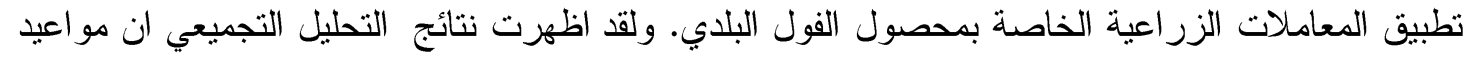

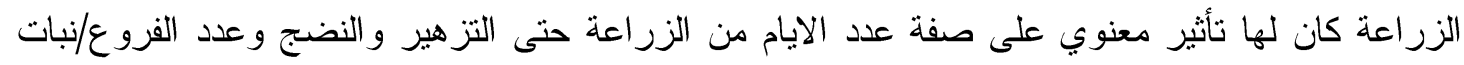
ومحصول البذور. كما اظهرت النتائج وجود فروق معنوية بين الثمانية تر اكيب وراثية في صفات عندئ عدد الايام

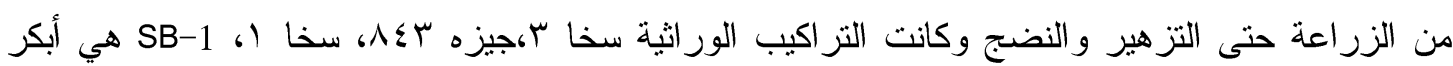
التز اكيب الور اثية في كلا من ملوى وسدس على ولى الترتيب. و اعطت التز اكيب الور اثية اعلى محصول بذور/فدان في الميعاد الثاني (ا نوفمبر) بالمقارنة بالميعادين

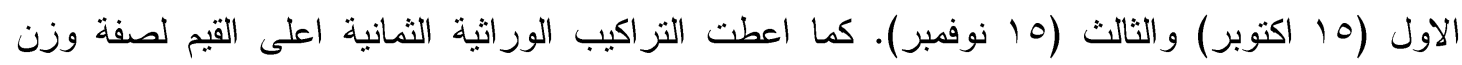

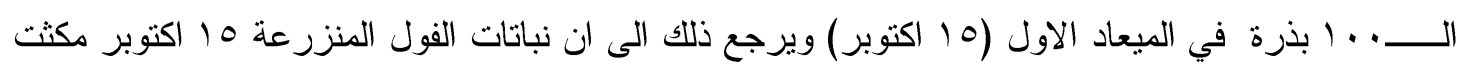

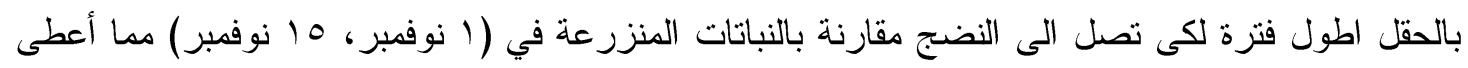

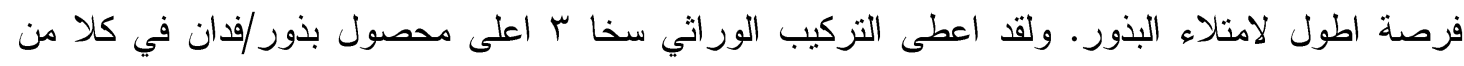

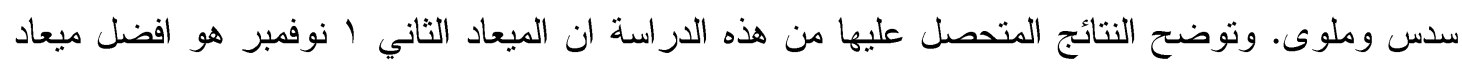

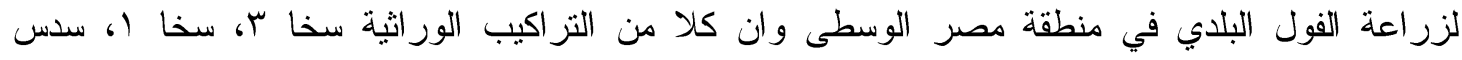
9 ايمكن ان يكونو ا اكثر تأقلماً مع مصر الوسطى. 